Volume 10, No.3, May - June 2021

International Journal of Advanced Trends in Computer Science and Engineering

Available Online at http://www.warse.org/IJATCSE/static/pdf/file/ijatcse1031032021.pdf

https://doi.org/10.30534/ijatcse/2021/1041032021

\title{
Operating Systems for Ethical Hackers - A Platform Comparison of Kali Linux and Parrot OS
}

\author{
Syed Zain ul Hassan ${ }^{1}$, Zainab Muzaffar ${ }^{2}$, Saleem Zubair Ahmad ${ }^{3}$ \\ ${ }^{1,2,3}$ Department of Software Engineering, Superior University Lahore, Pakistan. \\ ${ }^{1}$ E-mail: zain.ravian@gmail.com, ${ }^{2}$ E-mail: zainabmuzaffar228@gmail.com, \\ ${ }^{3}$ E-mail: Saleem.zubair@ superior.edu.pk
}

\begin{abstract}
Many operating systems are used for ethical hacking, which has emerged over the years. These operating systems have multiple tools and features to encounter malicious attacks performed by hackers. This study aims to discuss the benefits of various operating systems used for ethical hacking and to present a platform comparison study of two well-known Debian-derived Linux distributions used for ethical hacking, namely Kali Linux and Parrot OS. These tools and features assist ethical hackers in determining which operating system is best for penetration testing. In this paper, we will explore what penetration testing is, why we use this testing technique and how to secure the computer and the network from cyber-attacks using different ethical hacking operating systems. The paper deals with a qualitative analysis of the tools and features to deeply analyze some of their metrics which have been common in these operating systems. This paper will help ethical hackers to nail down the operating systems that are most suitable for them.
\end{abstract}

Keywords: Operating Systems, Kali Linux, Parrot OS, Penetration testing, Kali vs parrot, Ethical hacking OS, Cyber Security.

\section{INTRODUCTION}

An operating system is a type of software that has a wide range of definitions and is used to connect human commands to hardware responses [1]. Computers are now considered necessary, from the young to the elderly, from students to business executives. Every year, the number of computer users increases dramatically. The rapid increase in the number of computer users per year raises security concerns. Now, computer security has become critical and attackers are always searching for opportunities and vulnerabilities to gain access to others' personal data.

Penetration testing is a set of activities used to find and exploit security flaws in a computer system [2]. It assists in determining the effectiveness (or ineffectiveness) of the security measures put in place.
Vulnerability assessment's main goal is to find security issues in a controlled environment so that they may be corrected before they are exploited by unauthorized people. Computing system specialists employ penetration testing to resolve issues that appear during vulnerability assessments, with a focus on

high-severity defects. Penetration testing is a vital tool for ensuring the security of a system.

Our paper focuses on two well-known Ethical Hacking operating systems that run on machines to perform penetration testing tasks.

We have discussed a platform comparison between Parrot OS and Kali Linux. The comparison of different OSs in terms of their tools and features is needed to provide details on the advantages and disadvantages of both types of OS vis-à-vis their strengths and weaknesses.

\subsection{Operating Systems for Ethical Hackers}

This research presents different operating systems to be used for Ethical Hacking. Everything is open-source, free, and based on the Linux kernel, with a variety of hacking tools thrown in for good measure. Other Linux distributions are used for ethical hacking, in addition to the Kali distribution and the Parrot OS, which are the most common ones.

\subsubsection{Kali Linux [3]}

Kali Linux is a widely used open-source security operating system for penetration testing. There are vast array pre-installed penetration testing tools to perform a variety of data security functions, Penetration Testing, Security Analysis, Forensics, and Reverse Engineering are just a few examples.

\subsubsection{Parrot OS [4]}

Parrot OS is a new version of Linux that comes with several tools for penetration testing. Lightweight with dedicated CDNs. tools such as Anon Surf, Onion Share, TOR, I2P, etc. Parrot Security OS is a hacking distribution that is still in its infancy. 
Syed Zain ul Hassan et al., International Journal of Advanced Trends in Computer Science and Engineering, 10(3), May - June 2021, 2226 - 2233

\subsubsection{Backbox [5]}

The most well-known research techniques are aimed at a broad range of objectives, including web application analysis, network analysis, stress checks, sniffing, vulnerability evaluation, computer forensic analysis, automotive, and exploitation. Backbox is an Ubuntu-based penetration testing and vulnerability assessment distribution. It has its own software repository, which includes the most recent secure versions of many device and network analysis toolkits, as well as the most widely used ethical hacking tools. Backbox is a minimalist desktop environment that runs on the XFCE (XForms Popular Environment). It produces work that is fast, effective, and adaptable.

\subsubsection{BlackArch [6]}

BlackArch is a comprehensive Linux system for penetration testers and security researchers. It is based on Arch Linux and users can install individual or group BlackArch components directly on top of it. The repository contains 2668 penetration and security tools. Automation, mobile tools \& networking.

The toolset is available as an unauthorized Arch Linux user's repository, allowing you to install BlackArch on top of an existing Arch Linux system. Individual packages or categories of packages can be installed.

\subsubsection{Fedora Security Lab [7]}

Security auditing, forensics, system rescue, and education on security testing methods. Fedora Security Spin is a Fedora version built for security testing and auditing, as well as for educational purposes.

\subsubsection{Dracos Linux [8]}

Dracos Linux is a penetration testing operating system that is open source. Information collecting, forensics, virus analysis, access management, and reverse engineering are just a few of the pen test tools included. Forensics, information gathering $\&$ malware analysis. Having three main directories attack, defense and forensics.

\subsubsection{Bugtraq OS [9]}

Bugtraq is a software distribution that provides a wide variety of penetration testing, forensic, and laboratory resources. It runs on Ubuntu, Debian, and OpenSUSE and comes with the XFCE, GNOME, and KDE desktop environments. Penetration testing software, mobile forensics, and malware testing facilities, as well as Bugtraq-developed solutions, can all be found on Bugtraq.

\subsubsection{CAINE [10]}

CAINE Linux is the basis for Pentoo Linux. It's a security and penetration testing delivery that comes as a live $\mathrm{CD}$ with persistence. Pentoo uses the XFCE desktop environment, which provides a range of specialized tools and kernel features.

\subsubsection{Samurai Web Testing Framework [11]}

VMWare supports the Samurai Web Testing Framework as a virtual machine. Perform pen-testing and website attack tools. The Samurai Web Testing Framework was created specifically for web penetration testing. Another distinction from previous distributions is that it is available as a virtual machine, which is compatible with virtual boxes and VMWare. The Samurai Web Testing Platform is a free and open-source framework for testing and targeting websites that is based on Ubuntu.

\subsubsection{Network Security Toolkit [12]}

It performs regular security checks and network traffic monitoring tasks. Monitoring of virtual machines on a virtual server. The Network Security Toolkit is a Fedora-based bootable Live ISO (Live CD). It includes a large collection of open-source network security software, as well as an integrated web user interface for system and network management, navigation, automation, network control, and analysis, as well as the setup of many of the programs in the distribution.

\subsubsection{Demon Linux [13]}

Demon Linux is a modified Debian distribution for penetration testing. Hacking tools, VMWare \& LIVE with RAM/Squash FS. By pressing just one key, search or open anything.

\subsubsection{Arch Strike [14]}

Pen testing \& security layer, open-source tools for investigation. ArchStrike (previously Arch Assault) is an Arch Linux-based distribution for penetration testers and security professionals. It comes with all of the functionality of Arch Linux, as well as penetration testing and cyber security tools. On the Arch Strike website, tens of thousands of pieces of software and applications are organized into modular kit groups.

\subsubsection{Andrax [15]}

ANDRAX is a Desktop, Android, and ARM-based Advanced Penetration Testing Platform. Advanced Ethical Hacking and Penetration Testing on Several Platforms performs security checks on a wide range of devices (Desktop, Notebook, Android, Raspberry Pi). ANDRAX makes it possible for devices to be used as a weapon in Advanced Penetration Testing (APT) and Red Team operations.

\subsection{A Platform Comparison of Kali Linux and Parrot OS}

A platform comparison study of two well-known Debian-derived Linux distributions used for ethical hacking, which are Kali Linux and Parrot OS. 
Syed Zain ul Hassan et al., International Journal of Advanced Trends in Computer Science and Engineering, 10(3), May - June 2021, 2226 - 2233

\subsubsection{Kali Linux}

Kali Linux is an open-source Linux distribution based on Debian that is designed for various information security tasks like penetration testing, security analysis, computer forensics, and reverse engineering [3].Kali Linux is the most effective and widely used penetration testing tool in the world, with penetration testers, forensics experts, reverse engineers, and vulnerability assessors all using it. A large number of tools and utilities are included in the Kali Linux penetration testing platform. Kali Linux enables security and IT experts to examine the security of their systems from data collection to final reporting. Kali Linux comes with a variety of tools that fall into many categories, including [3]:

\subsubsection{Information Gathering}

The act of gathering various types of information against a targeted victim. Table 1 shows 67 different tools used in Kali Linux for gathering information.

Table 1. Information Gathering Tools [16]

\begin{tabular}{|l|l|l|l|l|l|}
\hline Sr. & Tools & Sr. & Tools & Sr. & Tools \\
\hline 1 & Ace-voip & 24 & Amap & 47 & Nbtscan-unixwiz \\
\hline 2 & Automater & 25 & APT2 & 48 & Bing-ip2hosts \\
\hline 3 & CDPSnarf & 26 & iSMTP & 49 & $\begin{array}{l}\text { copy-router-confi } \\
\text { g }\end{array}$ \\
\hline 4 & Dnmap & 27 & Dnsenum & 50 & dnsmap \\
\hline 5 & Dnstracer & 28 & Dnswalk & 51 & DotDotPwn \\
\hline 6 & EnumIAX & 29 & braa & 52 & Faraday \\
\hline 7 & Firewalk & 30 & Fragroute & 53 & fragrouter \\
\hline 8 & GoLismero & 31 & Goofile & 54 & Cisco-torch \\
\hline 9 & InSpy & 32 & InTrace & 55 & Metagoofil \\
\hline 10 & $\begin{array}{l}\text { Maltego } \\
\text { Teeth }\end{array}$ & 33 & Masscan & 56 & EyeWitness \\
\hline 11 & sslstrip & 34 & Nikto & 57 & Ident-user-enum \\
\hline 12 & URLCrazy & 35 & P0f & 58 & smtp-user-enum \\
\hline 13 & SET & 36 & SMBMap & 59 & SSLsplit \\
\hline 14 & SPARTA & 37 & Sslcaudit & 60 & THC-IPV6 \\
\hline 15 & SSLyze & 38 & Sublist3r & 61 & Unicornscan \\
\hline 16 & TLSSLed & 39 & Twofi & 62 & Xplico \\
\hline 17 & Wireshark & 40 & WOL-E & 63 & snmp-check \\
\hline 18 & Arp-scan & 41 & $\begin{array}{l}\text { Ghost } \\
\text { Phisher }\end{array}$ & 64 & DNSRecon \\
\hline 19 & CaseFile & 42 & hping3 & 65 & theHarvester \\
\hline 20 & DMitry & 43 & lbd & 66 & Enum4linux \\
\hline 21 & Parsero & 44 & Miranda & 67 & $\begin{array}{l}\text { OSR } \\
\text { Framework }\end{array}$ \\
\hline 22 & Nmap & 45 & Ntop & & \\
\hline 23 & Recon-ng & 46 & Fierce & & \\
\hline & & & & \\
\hline
\end{tabular}

\subsubsection{Vulnerability Analysis}

Vulnerability analysis entails discovering, assessing the severity of, and prioritizing any security issues before they are exploited by bad actors. Table 2 shows 27 different tools used in Kali Linux for Vulnerability Analysis.
Table 2. Vulnerability Analysis Tools [16]

\begin{tabular}{|l|l|l|l|l|l|}
\hline Sr. & Tools & Sr. & Tools & Sr. & Tools \\
\hline 1 & BBQSQL & 10 & BED & 19 & $\begin{array}{l}\text { cisco-auditing- } \\
\text { tool }\end{array}$ \\
\hline 2 & Cisco-ocs & 11 & openvas & 20 & $\begin{array}{l}\text { unix-privesc-ch } \\
\text { eck }\end{array}$ \\
\hline 3 & SidGuesser & 12 & sqlsus & 21 & jSQL Injection \\
\hline 4 & Nmap & 13 & ohrwurm & 22 & Powerfuzzer \\
\hline 5 & HexorBase & 14 & sfuzz & 23 & $\begin{array}{l}\text { copy-router-con } \\
\text { fig }\end{array}$ \\
\hline 6 & Sqlmap & 15 & Sqlninja & 24 & SIPArmyKnife \\
\hline 7 & THC-IPV6 & 16 & Yersinia & 25 & Tnscmd10g \\
\hline 8 & Cisco-torch & 17 & Lynis & 26 & DotDotPwn \\
\hline 9 & Doona & 18 & Oscanner & 27 & $\begin{array}{l}\text { cisco-global- } \\
\text { exploiter }\end{array}$ \\
\hline
\end{tabular}

\subsubsection{Exploitation Tools}

Exploitation is a piece of coded software or a script that allows hackers to gain control of a system by exploiting its flaws. Table 3 shows 21 different tools used in Kali Linux for Exploitation Tools.

Table 3. Exploitation Tools [16]

\begin{tabular}{|c|l|c|l|c|l|}
\hline Sr. & Tools & Sr. & Tools & Sr. & Tools \\
\hline 1 & Armitage & 8 & BeEF & 15 & $\begin{array}{l}\text { Backdoor } \\
\text { Factory }\end{array}$ \\
\hline 2 & cisco-torch & 9 & cisco-ocs & 16 & $\begin{array}{l}\text { cisco-auditing- } \\
\text { tool }\end{array}$ \\
\hline 3 & crackle & 10 & exploitdb & 17 & $\begin{array}{l}\text { jboss- } \\
\text { autopwn }\end{array}$ \\
\hline 4 & $\begin{array}{l}\text { Maltego } \\
\text { Teeth }\end{array}$ & 11 & MSFPC & 18 & $\begin{array}{l}\text { Metasploit } \\
\text { Framework }\end{array}$ \\
\hline 5 & SET & 12 & ShellNoob & 19 & RouterSploit \\
\hline 6 & Yersinia & 13 & $\begin{array}{l}\text { THC-IPV } \\
6\end{array}$ & 20 & $\begin{array}{l}\text { Linux Exploit } \\
\text { Suggester }\end{array}$ \\
\hline 7 & Commix & 14 & sqlmap & 21 & $\begin{array}{l}\text { cisco-global-ex } \\
\text { ploiter }\end{array}$ \\
\hline
\end{tabular}

\subsubsection{Wireless Attacks}

A harmful activity against wireless system information is referred to as a wireless attack. Table 4 shows 54 different tools used in Kali Linux for Wireless Attacks.

Table 4. Wireless Attacks Tools [16]

\begin{tabular}{|c|l|c|l|l|l|}
\hline Sr. & Tools & Sr. & Tools & Sr. & Tools \\
\hline 1 & Airbase-ng & 19 & Bully & 37 & $\begin{array}{l}\text { Airdecap-ng } \\
\text { and } \\
\text { Airdecloak-ng }\end{array}$ \\
\hline 2 & airgraph-ng & 20 & Airmon-ng & 38 & Airodump-ng \\
\hline 3 & Airolib-ng & 21 & Airserv-ng & 39 & Airtun-ng \\
\hline 4 & Besside-ng & 22 & Bluelog & 40 & BlueMaho \\
\hline 5 & BlueRanger & 23 & Bluesnarfer & 41 & Aircrack-ng \\
\hline
\end{tabular}


Syed Zain ul Hassan et al., International Journal of Advanced Trends in Computer Science and Engineering, 10(3), May - June 2021, 2226 - 2233

\begin{tabular}{|c|l|c|l|c|l|}
\hline 6 & crackle & 24 & Easside-ng & 42 & eapmd5pass \\
\hline 7 & mfoc & 25 & $\begin{array}{l}\text { Ghost } \\
\text { Phisher }\end{array}$ & 43 & GISKismet \\
\hline 8 & gr-scan & 26 & Spooftooph & 44 & $\begin{array}{l}\text { Packetforge- } \\
\text { ng }\end{array}$ \\
\hline 9 & KillerBee & 27 & Pyrit & 45 & makeivs-ng \\
\hline 10 & mfcuk & 28 & mfterm & 46 & $\begin{array}{l}\text { FreeRADIUS- } \\
\text { WPE }\end{array}$ \\
\hline 11 & mdk3 & 29 & PixieWPS & 47 & $\begin{array}{l}\text { airodump-ng-o } \\
\text { ui-update }\end{array}$ \\
\hline 12 & redfang & 30 & $\begin{array}{l}\text { RTLSDR } \\
\text { Scanner }\end{array}$ & 48 & hostapd-wpe \\
\hline 13 & Gqrx & 31 & Wifi Honey & 49 & wifiphisher \\
\hline 14 & Wifite & 32 & wpaclean & 50 & Aireplay-ng \\
\hline 15 & Kismet & 33 & coWPAtty & 51 & kalibrate-rtl \\
\hline 16 & Asleap & 34 & Tkiptun-ng & 52 & $\begin{array}{l}\text { Fern Wifi } \\
\text { Cracker }\end{array}$ \\
\hline 17 & Bluepot & 35 & Wifitap & 53 & Multimon-NG \\
\hline 18 & Reaver & 36 & ivstools & 54 & Wesside-ng \\
\hline
\end{tabular}

\subsubsection{Forensics Tools}

A harmful activity against wireless system information is referred to as a wireless attack. Table 5 shows 23 different tools used in Kali Linux for Wireless Attacks.

Table 5. Forensics Tools [16]

\begin{tabular}{|c|l|c|l|c|l|}
\hline Sr. & Tools & Sr. & Tools & Sr. & Tools \\
\hline 1 & Binwalk & 9 & p0f & 17 & Capstone \\
\hline 2 & Cuckoo & 10 & dc3dd & 18 & ddrescue \\
\hline 3 & diStorm3 & 11 & Galleta & 19 & extundelete \\
\hline 4 & Xplico & 12 & peepdf & 20 & $\begin{array}{l}\text { iPhone Backup } \\
\text { Analyzer }\end{array}$ \\
\hline 5 & pdf-parser & 13 & pdfid & 21 & bulk-extractor \\
\hline 6 & RegRipper & 14 & Volatility & 22 & Dumpzilla \\
\hline 7 & chntpw & 15 & Foremost & 23 & Guymager \\
\hline 8 & DFF & 16 & pdgmail & & \\
\hline
\end{tabular}

\subsubsection{Web Applications}

Kali uses the Web applications category to test/penetrate web applications. Table 6 shows 43 different tools used in Kali Linux for Web Applications.

Table 6. Web Applications tools [16]

\begin{tabular}{|c|l|c|l|c|l|}
\hline $\begin{array}{c}\text { Sr } \\
.\end{array}$ & Tools & $\begin{array}{l}\text { Sr } \\
.\end{array}$ & Tools & Sr. & Tools \\
\hline 1 & apache-users & 16 & Arachni & 31 & DAVTest \\
\hline 2 & Burp Suite & 17 & CutyCapt & 32 & fimap \\
\hline 3 & DIRB & 18 & DirBuster & 33 & hURL \\
\hline 4 & Gobuster & 19 & Grabber & 34 & Maltego Teeth \\
\hline
\end{tabular}

\begin{tabular}{|c|l|c|l|c|l|}
\hline 5 & joomscan & 20 & $\begin{array}{l}\text { jSQL } \\
\text { Injection }\end{array}$ & 35 & Parsero \\
\hline 6 & PadBuster & 21 & Paros & 36 & Recon-ng \\
\hline 7 & Powerfuzzer & 22 & $\begin{array}{l}\text { ProxyStrik } \\
\text { e }\end{array}$ & 37 & sqlsus \\
\hline 8 & sqlmap & 23 & Sqlninja & 38 & WebScarab \\
\hline 9 & Uniscan & 24 & w3af & 39 & Wfuzz \\
\hline 10 & WebSlayer & 25 & WebSploit & 40 & zaproxy \\
\hline 11 & WPScan & 26 & XSSer & 41 & Webshag \\
\hline 12 & $\begin{array}{l}\text { BlindElephan } \\
\text { t }\end{array}$ & 27 & Nikto & 42 & WhatWeb \\
\hline 13 & deblaze & 28 & plecost & 43 & jboss-autopwn \\
\hline 14 & FunkLoad & 29 & Skipfish & & \\
\hline 15 & ua-tester & 30 & BBQSQL & & \\
\hline
\end{tabular}

\subsubsection{Stress Testing}

Stressing tools are used to stress tests for various applications in order to take suitable preventative steps in the future. Table 7 shows 14 different tools used in Kali Linux for Stress Testing.

Table 7. Stress Testing tools [16]

\begin{tabular}{|c|l|c|l|c|l|}
\hline Sr. & Tools & Sr. & Tools & Sr. & Tools \\
\hline 1 & DHCPig & 6 & t50 & 11 & FunkLoad \\
\hline 2 & inviteflood & 7 & mdk3 & 12 & ipv6-toolkit \\
\hline 3 & rtpflood & 8 & iaxflood & 13 & $\begin{array}{l}\text { THC-SSL- } \\
\text { DOS }\end{array}$ \\
\hline 4 & THC-IPV6 & 9 & Termineter & 14 & $\begin{array}{l}\text { SlowHTTP } \\
\text { Test }\end{array}$ \\
\hline 5 & Reaver & 10 & Inundator & & \\
\hline
\end{tabular}

\subsubsection{Sniffing \& Spoofing}

Sniffing and spoofing entails wiretapping the network and monitoring all traffic entering and exiting it. Table 8 shows 33 different tools used in Kali Linux for Sniffing \& Spoofing.

Table 8. Sniffing \& Spoofing Tools [16]

\begin{tabular}{|c|l|c|l|c|l|}
\hline Sr. & Tools & Sr. & Tools & Sr. & Tools \\
\hline 1 & bettercap & 12 & Burp Suite & 23 & DNSChef \\
\hline 2 & fiked & 13 & $\begin{array}{l}\text { hamster-si } \\
\text { dejack }\end{array}$ & 24 & HexInject \\
\hline 3 & iaxflood & 14 & inviteflood & 25 & iSMTP \\
\hline 4 & isr-evilgrade & 15 & mitmproxy & 26 & ohrwurm \\
\hline 5 & protos-sip & 16 & rebind & 27 & responder \\
\hline 6 & rtpbreak & 17 & $\begin{array}{l}\text { rtpinsertso } \\
\text { und }\end{array}$ & 28 & rtpmixsound \\
\hline 7 & sctpscan & 18 & $\begin{array}{l}\text { SIPArmyK } \\
\text { nife }\end{array}$ & 29 & SIPp \\
\hline 8 & SIPVicious & 19 & SniffJoke & 30 & SSLsplit \\
\hline 9 & sslstrip & 20 & THC-IPV6 & 31 & VoIPHopper \\
\hline 10 & WebScarab & 21 & Wifi Honey & 32 & Wireshark \\
\hline 11 & xspy & 22 & Yersinia & 33 & zaproxy \\
\hline
\end{tabular}


Syed Zain ul Hassan et al., International Journal of Advanced Trends in Computer Science and Engineering, 10(3), May - June 2021, 2226 - 2233

\subsubsection{Password Attacks}

When a hacker attempts to steal your password, this is known as a password attack. Table 9 shows 38 different tools used in Kali Linux for Password Attacks.

Table 9. Password Attacks Tools [16]

\begin{tabular}{|c|l|c|l|c|l|}
\hline Sr. & Tools & Sr. & Tools & Sr. & Tools \\
\hline 1 & BruteSpray & 14 & Burp Suite & 27 & CeWL \\
\hline 2 & chntpw & 15 & $\begin{array}{l}\text { cisco-auditi } \\
\text { ng-tool }\end{array}$ & 28 & CmosPwd \\
\hline 3 & creddump & 16 & crowbar & 29 & crunch \\
\hline 4 & findmyhash & 17 & $\begin{array}{l}\text { gpp-decryp } \\
\text { t }\end{array}$ & 30 & $\begin{array}{l}\text { hash-identifi } \\
\text { er }\end{array}$ \\
\hline 5 & Hashcat & 18 & HexorBase & 31 & THC-Hydra \\
\hline 6 & $\begin{array}{l}\text { John the } \\
\text { Ripper }\end{array}$ & 19 & Johnny & 32 & keimpx \\
\hline 7 & $\begin{array}{l}\text { Maltego } \\
\text { Teeth }\end{array}$ & 20 & $\begin{array}{l}\text { Maskproce } \\
\text { ssor }\end{array}$ & 33 & multiforcer \\
\hline 8 & Ncrack & 21 & $\begin{array}{l}\text { oclgausscra } \\
\text { ck }\end{array}$ & 34 & ophcrack \\
\hline 9 & PACK & 22 & patator & 35 & $\begin{array}{l}\text { phrasendresc } \\
\text { her }\end{array}$ \\
\hline 10 & polenum & 23 & $\begin{array}{l}\text { RainbowCr } \\
\text { ack }\end{array}$ & 36 & rcracki-mt \\
\hline 11 & RSMangler & 24 & SecLists & 37 & SQLdict \\
\hline 12 & $\begin{array}{l}\text { Statsprocesso } \\
\text { r }\end{array}$ & 25 & $\begin{array}{l}\text { THC-pptp- } \\
\text { bruter }\end{array}$ & 38 & TrueCrack \\
\hline 13 & WebScarab & 26 & wordlists & & zaproxy \\
\hline
\end{tabular}

\subsubsection{Maintaining Access}

Maintaining Access is a phase of the pentest cycle with a very specific goal: to allow the pentester to stay in the targeted systems until he obtains what he perceives to be important information. Table 10 shows 17 different tools used in Kali Linux for Maintaining Access.

Table 10. Maintaining Access Tools [16]

\begin{tabular}{|c|l|c|l|c|l|}
\hline Sr. & Tools & Sr. & Tools & Sr. & Tools \\
\hline 1 & CryptCat & 7 & Cymothoa & 13 & dbd \\
\hline 2 & dns2tcp & 8 & $\begin{array}{l}\text { HTTPTun } \\
\text { nel }\end{array}$ & 14 & Intersect \\
\hline 3 & Nishang & 9 & polenum & 15 & PowerSploit \\
\hline 4 & pwnat & 10 & RidEnum & 16 & sbd \\
\hline 5 & shellter & 11 & U3-Pwn & 17 & Webshells \\
\hline 6 & Weevely & 12 & Winexe & & \\
\hline
\end{tabular}

\subsubsection{Reverse Engineering}

The act of deconstructing a thing to see how it works is known as reverse engineering. Table 11 shows 11 different tools used in Kali Linux for Reverse Engineering.
Table 11. Reverse Engineering Tools [16]

\begin{tabular}{|c|l|c|l|c|l|}
\hline Sr. & Tools & Sr. & Tools & Sr. & Tools \\
\hline 1 & apktool & 5 & dex2jar & 9 & diStorm3 \\
\hline 2 & edb-debugger & 6 & jad & 10 & javasnoop \\
\hline 3 & JD-GUI & 7 & OllyDbg & 11 & smali \\
\hline 4 & Valgrind & 8 & YARA & & \\
\hline
\end{tabular}

\subsubsection{Reporting Tools}

It's made to make data consolidation, querying, external command execution, and report production simple and straightforward. Table 12 shows 10 different tools used in Kali Linux for Reporting Tools.

Table 12. Reporting Tools [16]

\begin{tabular}{|c|l|c|l|c|l|}
\hline Sr. & Tools & Sr. & Tools & Sr. & Tools \\
\hline 1 & CaseFile & 5 & cherrytree & 8 & CutyCapt \\
\hline 2 & dos2unix & 6 & Dradis & 9 & MagicTree \\
\hline 3 & Metagoofil & 7 & Nipper-ng & 10 & pipal \\
\hline 4 & RDPY & & & & \\
\hline
\end{tabular}

\subsubsection{Hardware Hacking}

Hardware hacking refers to changing an existing piece of electronics to use it in ways it wasn't designed for. Table 13 shows 6 different tools used in Kali Linux for Hardware Hacking Tools.

Table 13. Hardware Hacking [16]

\begin{tabular}{|l|l|l|l|l|l|}
\hline Sr. & Tools & Sr. & Tools & Sr. & Tools \\
\hline 1 & android-sdk & 3 & apktool & 5 & Arduino \\
\hline 2 & dex2jar & 4 & Sakis3G & 6 & smali \\
\hline
\end{tabular}

\subsubsection{Parrot OS}

Parrot is a global group of developers and security experts who collaborate to establish a common framework of tools to make their jobs simpler, more standardized, and safer. Parrot OS, Parrot Security's flagship product, is a Debian-based GNU/Linux distribution built with security and privacy in mind [4]. It includes a complete portable laboratory for all types of cyber security operations, from pen testing to digital forensics and reverse engineering, as well as everything you'll need to write your own software or keep your data secure.

ParrotOS has all of the tools found in Kali Linux, as well as some of its own. Table 8 displays some Parrot OS in-built tools [4].

Table 8. Some Parrot OS in-built tools [3]

\begin{tabular}{|c|l|c|l|c|l|}
\hline Sr. & Tools & Sr. & Tools & Sr. & Tools \\
\hline 1 & I2P & 8 & AnonSurf & 15 & OnionShare \\
\hline \multirow{2}{*}{2} & $\begin{array}{l}\text { TOR (The } \\
\text { Onion } \\
\text { Routers) }\end{array}$ & 9 & $\begin{array}{l}\text { Electrum } \\
\text { Bitcoin } \\
\text { Wallet }\end{array}$ & 16 & Macchanger \\
\hline 3 & EtherApe & 10 & CUPP & 17 & Ricochet \\
\hline
\end{tabular}


Syed Zain ul Hassan et al., International Journal of Advanced Trends in Computer Science and Engineering, 10(3), May - June 2021, 2226 - 2233

\begin{tabular}{|c|l|c|l|c|l|}
\hline 4 & $\begin{array}{l}\text { Metasploit } \\
\text { Framework }\end{array}$ & 11 & $\begin{array}{l}\text { Kayak - } \\
\text { The Car } \\
\text { Hacking } \\
\text { Tool }\end{array}$ & 18 & $\begin{array}{l}\text { GPA - GNU } \\
\text { Privacy } \\
\text { Assistant }\end{array}$ \\
\hline 5 & Crunch & 12 & SQLMap & 19 & Nikto \\
\hline 6 & Bleachbit & 13 & Nmap & 20 & Aircrack-ng \\
\hline 7 & OPENVAS & 14 & Netcat & & \\
\hline
\end{tabular}

\section{LITERATURE REVIEW}

In this paper [17], Teddy Surya Gunawan et al. describe that computers and mobile devices connected through the internet exposed to many threats and exploitation [17]. With the help of penetration techniques offered by the Kali Linux operating system, it can be possible to minimize the threat level occurrence thought out the network. The authors explain some of the security parameters present in networks space, implementation of a security breach through penetration technique analysis the security vulnerability and auditing of security through kali Linux tools and techniques. The authors describe in a statically way that the Microsoft Windows OS (83.93\% use) and android operating system (69.68\% use) are most vulnerable/malicious because of widely used in the world that's why most of the attackers/hackers try to find a vulnerability in it to gain access to the system.

The penetration starts from identifying system vulnerabilities for this purpose the attacks/exploits tools used by authors in his research are (1) SQL Injection tool, (2) cross-site scripting tools, (3) Local/remote file inclusion tool, (4) Distributed Denial of services tool, (5) Man in middle attack tool, (6) Zero-day attack tool. In this paper, we explain more attacks/exploit tools in detail. The authors describe the role of security analysts in vulnerability assessment who collect data during an attack or any attempt of attack are going to perform by hacker, detect flaws in the system and maintain the record of the loopholes in the system through investigation. This investigation helps to find and report vulnerabilities in systems. The parameters of the investigation report include (1) Current situation of attack, (2) Impact of attack, (3) Evolution behavior of attack, (4) Forensic of deployed attack, (5) Prediction Information based on sources. Moreover, the author describes the role of the security audit process, in this process the finding and evaluation of vulnerability are made and the alternatives of this vulnerability are developed. Moreover, the author concludes by defining Kali Linux and the tools using for penetration testing.

The Linux Kernel is responsible for connecting the Android phones' hardware. The Linux Kernel is in charge of a number of assets, including memory, memory storage, memory distribution and de-allocation for the record structure, procedure booking, and system management [18].

\section{RESEARCH METHODOLOGY}

We conducted an SLR. In our investigation, we compared the platforms Kali Linux and Parrot OS in terms of tools and features. The objective of this research is to recognize a platform comparison of ParrotOS vs Kali Linux (Tools and Features) using an ethical hacking operating system. The study is based on a qualitative approach. The primary resources available include research papers, web blogs, website articles, and newspaper articles on the subject. To get a more detailed and in-depth understanding of this topic, the main goal was divided into the following research questions.

Q1. What are the hardware requirements for installing Kali Linux and Parrot OS?

Q2. Which operating system is best in terms of look and feel (user interface)?

Q3. What kinds of Operating System variants are there for Kali Linux and Parrot OS?

Q4. What kinds of penetration tools do Kali Linux and Parrot OS provides?

Q5. Which OS is better in term of performance comparison?

According to research questions, internet surfing is used to answer the above questions. The main reason for searching the internet is to search out the latest tools and features used by both operating systems.

Here are the highlights of a comparison of Linux distributions based on their hardware requirements, user interface, variations, tools, and performance.

\subsection{Hardware Requirements}

The minimum processor speed, memory, and disc space necessary to install Windows are among these criteria. Table 9 shows comparisons of both OS in terms of hardware requirements.

Table 9. Comparison in terms of hardware requirements [19].

\begin{tabular}{|l|l|}
\hline Kali Linux & Parrot OS \\
\hline $\begin{array}{l}\text { Kali Linux is } \\
\text { heavy-weight Operating } \\
\text { system }\end{array}$ & $\begin{array}{l}\text { Parrot OS is a light-weight } \\
\text { operating system. }\end{array}$ \\
\hline $\begin{array}{l}\text { Kali Linux makes use of } \\
\text { a number of tools that } \\
\text { require graphical } \\
\text { acceleration. }\end{array}$ & $\begin{array}{l}\text { The Parrot OS does not require } \\
\text { any graphical acceleration. }\end{array}$ \\
\hline $\begin{array}{l}\text { A minimum of 1GHZ } \\
\text { dual-core CPU is } \\
\text { needed. }\end{array}$ & $\begin{array}{l}\text { A minimum of 1GHZ dual-core } \\
\text { CPU is needed. }\end{array}$ \\
\hline $\begin{array}{l}\text { Minimum 2 GB of RAM } \\
\text { to install the } \\
\text { kali-Linux-default } \\
\text { metapackage and the } \\
\text { default Xfce4 desktop }\end{array}$ & $\begin{array}{l}\text { Minimum 256MB RAM for } \\
\text { i386 and 320MB RAM for } \\
\text { AMd64 architectures. } \\
\text { 512MB or more is } \\
\text { recommended. }\end{array}$ \\
$\begin{array}{l}\text { Both legacy and UEFI } \\
\text { boot modes are } \\
\text { supported. }\end{array}$ & $\begin{array}{l}\text { Both legacy and UEFI boot } \\
\text { modes are supported. }\end{array}$ \\
\hline $\begin{array}{l}\text { To install and start } \\
\text { working with Kali }\end{array}$ & $\begin{array}{l}\text { To install and start working } \\
\text { with Kali Linux, you'll need at }\end{array}$ \\
\hline
\end{tabular}


Syed Zain ul Hassan et al., International Journal of Advanced Trends in Computer Science and Engineering, 10(3), May - June 2021, 2226 - 2233

\begin{tabular}{|l|l|}
\hline $\begin{array}{l}\text { Linux, you'll need at } \\
\text { least 20GB of storage } \\
\text { space. }\end{array}$ & least 16GB of storage space. \\
\hline $\begin{array}{l}\text { After installation, Kali } \\
\text { Linux has a larger } \\
\text { deployment size. }\end{array}$ & $\begin{array}{l}\text { After installation, Parrot OS } \\
\text { has a smaller deployment size. }\end{array}$ \\
\hline $\begin{array}{l}\text { Both 32-bit and 64-bit } \\
\text { processors are } \\
\text { supported. }\end{array}$ & $\begin{array}{l}\text { The most recent version of } \\
\text { Parrot OS only works with } \\
\text { 64-bit processors. }\end{array}$ \\
\hline
\end{tabular}

\subsection{User Interfaces}

The operating system provides a user interface (UI), which is an environment in which the user interacts with the machine. Table 10 shows comparisons of both OS in terms of hardware user interface.

Table 10. Comparison in terms of user interfaces [19]

\begin{tabular}{|l|l|}
\hline \multicolumn{1}{|c|}{ Kali Linux } & \multicolumn{1}{c|}{ Parrot OS } \\
\hline $\begin{array}{l}\text { Its user interface is based } \\
\text { on the Gnome desktop } \\
\text { environment. }\end{array}$ & $\begin{array}{l}\text { The Ubuntu-Matte-Desktop- } \\
\text { Environment is used. }\end{array}$ \\
\hline $\begin{array}{l}\text { It has a simpler user } \\
\text { interface. }\end{array}$ & $\begin{array}{l}\text { It has much better user } \\
\text { interface. }\end{array}$ \\
\hline $\begin{array}{l}\text { It has hefty specifications } \\
\text { and is a little slow. }\end{array}$ & $\begin{array}{l}\text { It's tiny and compact, and it } \\
\text { doesn't lag much. }\end{array}$ \\
\hline
\end{tabular}

\subsection{Variations}

The comparison between different variations of both operating systems is shown in Table 11.

Table 11. Comparison in terms of Variations [19]

\begin{tabular}{|l|l|}
\hline \multicolumn{1}{|c|}{ Kali Linux } & \multicolumn{1}{c|}{ Parrot OS } \\
\hline Kali Lite Edition. & Parrot Sec OS Lite Edition. \\
\hline Kali Full Edition. & Parrot Sec OS Full Edition. \\
\hline $\begin{array}{l}\text { Kali armhf/armel (IoT } \\
\text { devices). }\end{array}$ & Parrot Sec OS Air Edition. \\
\hline $\begin{array}{l}\text { Kali Desktop Variation } \\
\text { (e17/KDE/Xfce). }\end{array}$ & Parrot Sec OS Studio Edition. \\
\hline
\end{tabular}

\subsection{Tools}

Table 12 shows a comparison of various tools for both operating systems.

Table 12. Comparison in terms of tools [20]

\begin{tabular}{|l|l|}
\hline \multicolumn{1}{|c|}{ Kali Linux } & \multicolumn{1}{|c|}{ Parrot OS } \\
\hline $\begin{array}{l}\text { Kali Linux offers a vast } \\
\text { array of inbuilt penetration } \\
\text { tools. }\end{array}$ & $\begin{array}{l}\text { Parrot OS offers a vast array } \\
\text { of inbuilt penetration tools. }\end{array}$ \\
\hline $\begin{array}{l}\text { It does not come with } \\
\text { built-in compilers or IDEs. }\end{array}$ & $\begin{array}{l}\text { It comes with a range of } \\
\text { compilers and IDEs } \\
\text { pre-installed. }\end{array}$ \\
\hline
\end{tabular}

\begin{tabular}{|l|l|}
\hline $\begin{array}{l}\text { It comes with all of the } \\
\text { required hacking tools. }\end{array}$ & $\begin{array}{l}\text { While it contains all of the } \\
\text { tools used in Kali, it also } \\
\text { adds its own. AnonSurf, } \\
\text { Wifiphisher, and Airgeddon } \\
\text { are only a few examples. }\end{array}$ \\
\hline $\begin{array}{l}\text { Support for penetration } \\
\text { testing tools only. No } \\
\text { dealing with inbuilt tools } \\
\text { for development purpose. }\end{array}$ & $\begin{array}{l}\text { A complete development } \\
\text { stack that comes } \\
\text { editors, languages, and tools. } \\
\text { Multimedia and office } \\
\text { packages are available. }\end{array}$ \\
\hline
\end{tabular}

\subsection{Performance}

Table 13 shows a comparison of the performance of both operating systems.

Table 13. Comparison in terms of performance [21]

\begin{tabular}{|l|l|}
\hline \multicolumn{1}{|c|}{ Kali Linux } & \multicolumn{1}{|c|}{ Parrot OS } \\
\hline $\begin{array}{l}\text { Kali is a little leggy, and } \\
\text { running it on a low-end } \\
\text { system can be a nightmare } \\
\text { when a brute-force attack }\end{array}$ & $\begin{array}{l}\text { It's really light and doesn't lag } \\
\text { much, because it can run on } \\
\text { low-end systems as well. }\end{array}$ \\
$\begin{array}{l}\text { is running in the } \\
\text { background while you're } \\
\text { doing anything else. }\end{array}$ & \\
\hline $\begin{array}{l}\text { Kali Linux has a large } \\
\text { user base and a vibrant } \\
\text { support community. Large } \\
\text { repository. }\end{array}$ & $\begin{array}{l}\text { The Parrot community is } \\
\text { rapidly growing and low } \\
\text { repository. }\end{array}$ \\
\hline
\end{tabular}

\section{RESULT AND DISCUSSION}

By study different comparison between Kali Linux and Parrot OS. We conclude the followings results:

- Both are helpful when it comes to penetration testing.

- Both are designed according to Debian guidelines.

- Both 32-bit and 64-bit architectures are supported.

When opposed to Kali Linux, ParrotOS wins when it comes to general resources and usable features. ParrotOS includes many of the resources found in Kali Linux, as well as some of its own. There are a few tools on ParrotOS that aren't available on Kali Linux. Let's take a look at a couple of them.

\subsection{Wifiphisher [22]}

When conducting Wi-Fi security testing, Wifiphisher is one of the tools needed. Clients can be targeted, and attacks can be carried out to easily introduce malware and other malicious software into the victim's network. It's a tool that can be tailored to easily capture and acquire all of the credentials required to penetrate the network in a systematic manner.

\subsection{AnonSurf [23]}

Anonymity is one of the most important requirements for hacking into anyone's architecture. When working on this, there is no ideal textbook procedure for absolutely being 
Syed Zain ul Hassan et al., International Journal of Advanced Trends in Computer Science and Engineering, 10(3), May - June 2021, 2226 - 2233

anonymous. Despite the fact that there are several choices, AnonSurf takes the lead because of its powerful ability to use Tor IPtables to anonymize an entire device. Tor is a package that comes preinstalled with Parrot OS and allows you to browse the internet anonymously.

\subsection{OnionShare [24]}

Onion Transmit is an open source that allows you to securely and anonymously share files of any size through the Tor network. It's extremely safe and simple to use; simply drag and drop your file onto the OnionShare. It will then construct a long random URL that the recipient can use to download the file using the TOR browser over the TOR network.

\section{CONCLUSIONS}

The goal of this paper is to discuss two Linux-based ethical hacking operating systems, which are Kali Linux and Parrot OS. This paper provides a detailed analysis of their tools and features. Multiple successful tools are integrated into both operating systems, which are specially created to carry out heterogeneous forms of attacks and find vulnerabilities by using penetration testing. Choosing an Operating System for ethical hacking is solely based on user preferences, customization, and system specifications. The purpose of using both operating systems is the same for penetration testing, but they have slightly different audiences. Kali Linux is more focused on security experts who want to use the OS for offensive purposes, whereas Parrot OS is more focused on privacy, anonymity, cryptography, security assessment and software development. Kali is resource intensive. To get the most out of Kali Linux, you should use high-spec hardware. If your hardware resources are limited, choose Parrot OS because it is lightweight and all of the tools run smoothly on low system specifications. In the next research work, we will discuss and compare more Linux variants used for ethical hacking.

\section{REFERENCES}

1. Alhassan, H., Bach, C.: Operating System and Decision Making. Presented at the April 3 (2014).

2. A. Bacudio, X. Yuan, B. Chu, and M. Jones, "An Overview of Penetration Testing," International Journal of Network Security \& Its Applications, vol. 3, pp. 19-38, Nov. 2011, doi: 10.5121/ijnsa.2011.3602.

3. "Kali Linux | Penetration Testing and Ethical Hacking Linux Distribution," Kali Linux. https://www.kali.org/ (accessed May 10, 2021).

4. "Parrot Security." https://www.parrotsec.org/ (accessed Mar. 31, 2021).

5. “Homepage," BackBox.org.https://www.backbox.org/ (accessed May 10, 2021).

6. "BlackArch Linux - Penetration Testing Distribution." https://blackarch.org/ (accessed May 10, 2021).
7. “SecurityLab.”https://labs.fedoraproject.org/en/ security/(accessed May 10, 2021).

8. "Dracos Linux." https://dracos-linux.org/ (accessed May 10, 2021).

9. “Bugtraq - ArchiveOS.” https://archiveos.org/bugtraq/ (accessed May 10, 2021).

10. "CAINE Live USB/DVD - computer forensics digital forensics." https://www.caine-live.net/ (accessed May 10, 2021).

11. "Samurai Web Testing Framework - SecTools Top Network Security Tools." https://sectools.org/tool/samurai/ (accessed May 10, 2021).

12. "Network Security Toolkit (NST 32)." https://www.networksecuritytoolkit.org/nst/index.html (accessed May 10, 2021).

13. "Demon Linux." https://www.demonlinux.com/ (accessed May 10, 2021).

14. “ArchStrike.” https://archstrike.org/ (accessed May 10, 2021).

15. "ANDRAX Hackers Platform."https://andrax.thecrackertechnology.com/(ac cessed May 10, 2021).

16. "Kali Linux Tools Listing." https://tools.kali.org/tools-listing (accessed Jun. 24, 2021).

17. T. Gunawan, M. Lim, N. Zulkurnain, and M. Kartiwi, "On the Review and Setup of Security Audit using Kali Linux," Indonesian Journal of Electrical Engineering and Computer Science, vol. 11, pp. 51-59, Jul. 2018, doi: 10.11591/ijeecs.v11.i1.pp51-59.

18. R. K. R. G, "Armoring Client and Servers Running on Linux Based Android Platform,” IJATCSE, vol. 8, no. 3 , pp. 479-486, Jun. 2019, doi: 10.30534/ijatcse/2019/22832019.

19. Parrot OS vs Kali Linux : which is best for Ethical Hacking, https://ethicalhackersacademy.com/blogs/ethical-hacker s-academy/parrot-os-vs-kali-linux

20. Difference between Kali Linux and Parrot OS, https://www.geeksforgeeks.org/difference-between-kalilinux-and-parrot-os/, (2020).

21. "Kali Linux vs Parrot Security OS: Operating System for Penetration Testing in a Nutshell | Hacker Noon."

https://hackernoon.com/operating-system-for-penetratio n-testing-in-a-nutshell-kali-linux-vs-parrot-security-os384809e7b7ae (accessed Jun. 24, 2021). 2021).

22. "GitHub - wifiphisher/wifiphisher: The Rogue Access Point Framework." https://github.com/wifiphisher/wifiphisher (accessed Jun. 21, 2021).

23. A. Aslam, "Anonsurf - Linux Hint." https://linuxhint.com/anonsurf/ (accessed Jun. 21, 2021).

24. “ OnionShare.” https://onionshare.org/ (accessed Jun. 24, 2021). 